\title{
Industrias culturales y crecimiento económico. Un modelo para el estudio del surgimiento de clusters creativos
}

\section{Cultural industries and economic growth. A model to study the emergence of creative clusters}

\author{
Ezequiel Avilés-OchoA \\ Paola M. Canizalez-Ramírez*
}

\begin{abstract}
The clear link between economy and culture revitalizes the interest in cultural industries due to its conceptualization and implementation. However, cultural industries lack the instruments corresponding to its perceptible economic participation. A schema of variables of flourishing industries and a model of measurement are proposed through theories of endogenous growth and new geography. It was found that cultural industries are agglomerated in creative clusters with a system of production of internal economies, whose drive depends on human capital, specialization, consumption, and related variety. As a corollary, the model determines that growth is in function of potentiating such factors in conditioning distinctive processes.
\end{abstract}

Keywords: cultural industries, cluster, creativity, economic growth.

\section{Resumen}

El vínculo manifiesto entre economía y cultura revitaliza el interés en industrias culturales dado su conceptualización e implementación, no obstante, carecen de instrumentos correspondientes a su participación económica perceptible. Al retomar teorías de crecimiento endógeno y nueva geografía económica se propone un esquema de variables de industrias florecientes y un modelo de medición. Se encontró que éstas se aglomeran en clusters creativos con un sistema de producción de economías internas, cuya motricidad depende del capital humano, especialización, consumo y variedad relacionada. Como corolario, el modelo determina que el crecimiento está en función de potencializar tales factores en procesos distintivos condicionantes.

Palabras clave: industrias culturales, cluster, creatividad, crecimiento económico.

*Universidad de Occidente, México. Correos-e: ezequiel.aviles@udo.mx, paola.canizalez@ udo.mx 


\section{Introducción}

El concepto de cultura vinculado con economía constituye una de las realidades más complejas en las ciencias sociales. A fines de los ochenta la perspectiva de la cultura como un bien sujeto de comercialización en masa y la disrupción entre ésta y la creatividad se acentuó, favoreciendo el surgimiento de industrias culturales, mismas que por sus particularidades generan diversas percepciones en forma, contexto y teoría. En los círculos académicos, el debate recurrente es la diferenciación entre las industriales y las creativas, afán que en la mayoría de los casos, carece de sentido experimental.

En años recientes, un cuerpo teórico alternativo se ordena apoyando la noción del estudio de la economía cultural; aporta resultados de tinte empírico al conjugar el escenario del desarrollo regional y las líneas de la nueva geografía económica (Scott, 2001; Markusen, 2006; Throsby, 2008a, y Kloosterman, 2010). La consecución de los objetivos particulares de esta investigación se inserta en esta corriente y considera enfatizarla en tres fases:

a) Inicialmente, se retoman las principales teorías del crecimiento económico, particularmente las de la corriente endógena y la nueva geografía económica para exponer que capital humano, ideas (creatividad) y tecnología (innovación) constituyen las variables motrices de estas industrias.

b) Posteriormente, los impulsos se despliegan en el espacio territorial, conformando economías de aglomeración. El sistema de producción se articula en distritos centrales de actividad innovadora, lo que geográficamente se expresa en la delimitación de un cluster creativo. Se retoman algunas premisas de la geografía económica (Krugman, 1991; Storper y Walker, 1989; Scott, 2000), para exponer que tales industrias revelan más que creatividad.

c) Finalmente, se expone una fase donde la explotación de su potencial creativo, sobrepasa los estándares del discurso crítico, al ser parte activa de la dinámica económica actual. A partir de la conjunción de la teoría endógena y los patrones de crecimiento geográfico, es posible crear un modelo que permita evaluar el impacto de una industria cultural floreciente en el crecimiento. Para clasificarla se toma en cuenta su actividad creativa, así como los bienes y servicios fabricados, los cuales conforman un vínculo entre producción y consumo de cultura al establecer mecanismos de retroalimentación y productividad.

El objetivo y aportación central del artículo es fusionar las variables distintivas de estas industrias en un modelo de crecimiento económico, orientado a organizar el sistema de producción creativo, intensificar los 
factores cinéticos, integrar los parámetros económicos perceptibles y los factores endógenos distintivos de cada cluster, para activar la causalidad del ciclo económico en virtud de la economía existente.

El argumento subyace en una discusión donde los análisis vertidos se remiten a cuestiones teóricas de índole relacional, enfocando la atención en aquellas industrias culturales donde la cadena de la creatividad, en su totalidad, tiene como objetivo principal la transmisión de conceptos estéticos o intelectuales con respuesta emocional y cognitiva, como el filme, artes escénicas, radiodifusión, publicación y redacción.

Se aprecia, sin embargo, que su clasificación incluye actividades de consumo indirecto, resultado de la actividad creativa-artística, cuyo propósito primario no es la transmisión de conceptos estéticos o intelectuales; un ejemplo son el diseńo, la moda, la arquitectura, la publicidad, entre otras. Su demanda dibuja una curva ascendente, la cual corresponde paralelamente al surgimiento de firmas especializadas y extendidas geográficamente. Evaluarlas, mediante instrumentos que valoren el impacto de la actividad productiva y los factores motores en aglomeraciones creativas, es una vía alterna y complementaria a las materias del crecimiento económico y competitividad regional.

\section{Industrias culturales: visión funcional para el crecimiento de regiones creativas}

Desde finales del siglo xx el perfil industrial de muchas economías se orientó en dirección de la economía cultural; en algunos países, ésta es reconocida como una de las actividades más dinámicas en cuanto a producción y empleo. Ello, señala Scott (2010), se debe al resurgimiento de la llamada nueva economía del capitalismo contemporáneo, la cual se distingue por una agregación de sectores de manufactura y servicios, cuyas características operacionales involucran un alto nivel organizacional y flexibilidad tecnológica, relaciones interfirmas de transacciones intensivas y la elaboración de productos de diseño especializado.

Importantes sectores de manufactura de alta tecnología, negocios y servicios financieros encajan en esta descripción (Lash y Urry, 1994; Pratt, 1997; Kloosterman, 2008). Todos ellos son objetos de intenso escrutinio por su impacto en la dinámica productiva y el progreso local.

En la también llamada economía creativa, una gran proporción de los recursos intelectuales y creativos del mundo se invierten en industrias basadas en la cultura, cuya producción intangible es tan real y considerable como la de otras; las industrias culturales son formas de producción y consumo que tienen en su centro un elemento expresivo o simbólico, 
la creatividad humana y la innovación a nivel individual y grupal son la clave que las impulsa (unESCO, 2013).

Su rápido crecimiento es resultado de la convergencia entre el orden económico y los sistemas de expresión cultural, atraen la atención de la política económica instrumentada en las naciones con mayor peso en el intercambio comercial. Así, de acuerdo con la Conferencia de las Naciones Unidas sobre Comercio y Desarrollo, UNCTAD (2009), el valor global de su mercado se valoró en 1.3 trillones de dólares en 2005, y desde 2000, crecen a un ritmo anual de más de 7\%. Se estima que en el 2005, las industrias culturales y creativas significaron más del $7 \%$ del Producto Interno Bruto (РIB) mundial; mientras que en 2009 registran un crecimiento de 1.7 trillones de dólares (UnEsCo, 2009).

Para la Organización para la Cooperación y el Desarrollo Económico, OCDE (2009), estas industrias son clave entre los países miembros, con un crecimiento anual que oscila entre $5 \%$ y $20 \%$. El comercio mundial en bienes y servicios creativos totalizó un récord de 624 billones de dólares en 2011, superando los 559.5 billones de dólares de 2010. El intercambio global en productos creativos se duplicó de 2002 a 2011, la tasa promedio anual de crecimiento fue de $8.8 \%$. Las exportaciones en países desarrollados aumentó a $12.1 \%$ anual en el mismo periodo, lo que alcanzó 227 billones de dólares en 2011, 50\% del total global (UNCTAD, 2013).

El protagonismo económico propicia que se desarrollen regiones orientadas a la especialización de productos y servicios culturales con nuevos patrones de competencia y formas de organización local. Al interior de esta causalidad se atrae la acción de fuerzas productivas distintivas, desplegando una economía de vocación diferente.

Para advertir tales efectos, la propuesta central del estudio retoma los parámetros teóricos del crecimiento endógeno y la nueva geografía económica, como instrumentos de análisis de los determinantes del ritmo de crecimiento y productividad entre regiones creativas, puesto que particularmente estas industrias destacan la intensificación del capital humano y los procesos de productividad e innovación.

$\mathrm{Al}$ respecto, Scott (2004) afirma que la nueva economía está representada por sectores como la manufactura de alta tecnología, productos de consumos neoartesanales y diversos servicios, los cuales tienen propensión a tomar la forma de una organización compleja de redes de valor agregado. La propensión de las firmas de los sectores de productos culturales es converger en clusters espaciales distintivos, lo cual es reflejo de una estructura organizacional en la que cada unidad de producción individual es atrapada en un amplio sistema de interacciones socioeconómicas.

Así, la aglomeración en clusters de competencia regional promueve desafíos relacionados a la reproducción de factores de crecimiento en 
espacios geográficos con industrias florecientes, creando un fenómeno multifacético que incorpora no sólo cúmulos de firmas especializadas y complementarias, sino otros elementos tales como mano de obra calificada y no calificada, redes sociales e instituciones de orden colectivo (Scott, 2006).

Asimismo, la hipótesis sobre la expansión de la productividad de clusters creativos conlleva a la propuesta de un modelo sistémico de industrias culturales, el cual centra su atención en el análisis de las variables propulsoras relacionadas a las economías internas que componen cada aglomeración.

\subsection{La imagen dialéctica de industrias culturales}

En 1947, Horkheimer y Adorno acuñaron el término industria cultural como una crítica hacia la inherente mercantilización en masa de la cultura (Adorno, 1991). Desde entonces, existen nociones que conceptualizan, critican y aportan líneas de discusión en la misma dirección, tendencia que propone ambivalencia y correlación teórica.

De forma somera, estas industrias representan uno de los segmentos más prolíficos y se identifican básicamente como proveedores de productos culturales. Estas implicaciones para Scott (2001) se manifiestan dado que la sociedad contemporánea se distingue por la convergencia evidente entre el dominio de lo económico, por un lado, y la extensión de lo cultural, por el otro. Lo que expone una economía cultural representada por sectores (equivalente a industrias de productos culturales) que elaboran bienes y servicios, cuyo significado subjetivo, o más estrechamente, el valor para el consumidor es alto en comparación con sus propósitos utilitarios.

Para Throsby (2001), la noción de cultura como economía lleva a la propuesta de que su producción y consumo se pueden situar dentro de un marco industrial, que los bienes y servicios culturales producidos y consumidos se pueden considerar como mercancías en los mismos términos que otros bienes originados en el sistema económico. En esta discusión, Pratt (2008) asume que tienen presencia económica y cultural, su importancia radica en la relación estrecha que mantienen con la producción; son uno de los motores potenciales para el crecimiento y la regeneración urbana; un ejemplo práctico de las complejas relaciones híbridas entre producción y consumo es lo simbólico y lo material.

La identificación de una industria cultural en el sistema económico se vincula al proceso de producción, consumo e intercambio de determinados artículos; sus productos se clasifican al diferenciar los bienes y servicios, los que suponen creatividad, incorporan cierto grado de propiedad inte- 
lectual y transmiten un significado simbólico (Throsby, 2008a). En esta dinámica, la economía se hace más diferenciada y los factores culturales ejercen importante influencia sobre los métodos de producción y los tipos de productos, mismos que se insertan en la competitividad del mercado mundial para responder a una demanda especializada en constante aumento. Entonces, la posición que mantienen en la economía se consolida, en razón del incremento en su intercambio comercial, los ajustes en las dinámicas de consumo y la creación de una oferta diferenciada en razón de innovación, tecnología y creatividad.

En tal aseveración, el valor cultural o el contenido de bienes y servicios producidos es lo que da a las industrias culturales su característica más distintiva. Distintos bienes o servicios tienen diferentes niveles de contenido cultural para su valor comercial; entre más pronunciado sea éste, cuanto más fuerte es la demanda de la industria que lo produce para ser considerada como industria cultural (Throsby, 2008b).

En otra vertiente, Hesmondhalgh (2002) plantea que más que otro tipo de producción, estas industrias están incluidas en la elaboración y circulación de bienes que tienen una influencia sobre el entendimiento del mundo. Así, se relacionan en la producción de significado social en forma de textos y símbolos; están conectadas, principalmente, con la organización y venta de un tipo particular de trabajo y son agentes de cambio económico, social y cultural.

Por su parte Markusen (2006: 6), las define como "aquellos establecimientos (lucrativos, no lucrativos y públicos) que producen bienes y servicios culturales". Las relaciona como un conjunto de sectores que incluye a las industrias manufactureras y de servicios, lo que constituye un fenómeno único y creciente en la cooperación regional y al interior de las economías nacionales.

En este contexto, su utilización como detonante de crecimiento revela la propuesta de que pueden ser definidas en términos concretos como un ensamble de sectores que ofrecen productos de servicios enfocados en el entretenimiento, edificación, información (Scott, 2010). Al respecto, Power y Scott (2004: 3-4), proponen tres características:

a) Están relacionadas de una manera u otra con la creación de contenido estético y semiótico; b) Son generalmente sujeto de los efectos de la Ley de Engels; es decir, en tanto se expande el ingreso disponible, el consumo de los productos culturales aumenta significativamente. Son bienes normales en todo momento y, c) Son frecuentemente sujeto de presiones competitivas, que obliga a las firmas individuales a aglomerarse en densos clusters especializados o distritos industriales, mientras que sus productos circulan con facilidad en los mercados globales. 
Tales condiciones se observan en gran parte de la esfera de actividad productiva, penetrada por tecnologías digitales y formas organizacionales flexibles, sostienen sectores que prosperan de manera diferente en materia de innovación continua, diversidad de productos y prestación de servicios personalizados. Es así que la economía cultural se expande, no sólo en función del crecimiento de un ingreso discrecional, sino como una expresión de la incursión de su valor dentro de las amplias esferas de la actividad productiva (Scott, 2004).

Entonces, dentro del sistema económico se reconocen nuevas formas de organización de la producción y nuevos sistemas localizados de actividad en torno a industrias culturales, mismas que son visiblemente conductores del desarrollo económico local, así, el florecimiento de sitios de producción cultural son estudiados intensamente por Whitt, 1987; Bianchini, 1993; O'connor, 1998; Landry, 2000; Throsby, 2001; Fuchs, 2002 (citados en Scott, 2004). Entre otras variantes, estos autores debaten sobre las potencialidades de la economía cultural en la creación de empleo y regeneración urbana en áreas rezagadas. Esto permite que tal fenómeno se valore desde una perspectiva teórica y empírica particular, lo que exige la necesidad de retomarla.

\section{Perspectiva funcional de la economía cultural para el crecimiento económico. Inclusión de la creatividad y el factor endógeno}

Considerables esfuerzos se enfocan en otorgarle carácter cuantitativo a las industrias culturales, los cuales se pierden, la mayoría de las veces, dadas las condiciones relativas a su definición. Throsby (2008a) señala que, aún si existiera un concepto general, los códigos ocupacionales e industriales a través del mundo hacen imposible establecer un parámetro de cuentas totalmente comparable. En la apreciación de Prince (2014), la instrumentalización de la cultura y la representación de sus aspectos locales para obtener logros económicos y sociales es una tendencia política conspicua mundial en las últimas décadas.

La evidencia publicada sobre su impacto, sugiere que éstas son importantes para la dinámica económica regional. Pratt (1997), por ejemplo, muestra para el caso de Gran Bretaña en 1991, que más de un millón de trabajadores ( $4.5 \%$ de la fuerza de trabajo total) son empleados en estas industrias. Asimismo, en 2001, sus resultados muestran que estas industrias en su mayoría se encuentran localizadas en grandes ciudades, en este caso Londres incuba 26.9\% del empleo de Gran Bretańa.

En otro estudio, a partir de la definición basada en categorías de estándares industriales, Scott (2004) indica que en Estados Unidos estas industrias significaron en 1992 más de tres millones de trabajadores, lo 
que representa $2.4 \%$ del total de la fuerza de trabajo; además, más de $50 \%$ se concentra en áreas metropolitanas con población de un millón o más, principalmente en Nueva York y Los Ángeles.

Así, entre los análisis empíricos que relacionan a los productos de industrias culturales con parámetros económicos, destacan los de Pratt (1997), Hesmondhalgh (2002), Scott (2004), Markusen (2006), Throsby (2008a), Gertler (2008) y Kloosterman (2010). Estos autores muestran que éstas constituyen un elemento proporcional moderno de los sistemas económicos nacionales, y son capaces de generar contribuciones medibles al empleo absoluto e ingreso.

En muchas áreas metropolitanas son el elemento principal de la economía, su rasgo distintivo es que se expanden con gran rapidez; empero, también numerosas pequeñas y medianas ciudades son lugares propicios de desarrollo económico cultural.

En análisis como los de Caves (2003) esta economía se caracteriza por transacciones y modelos de producción contractuales, con actividades en una red social de trabajadores creativos especializados. Estas características se vinculan con procesos productivos endógenos, evolución que requiere adaptación continua, innovación tecnológica, intercambio de información, productos diferenciados y regulación del mercado, lo cual otorga garantías a los procesos de crecimiento sustentable en razón de que los determinantes relacionados al aumento de la productividad y la expansión del ritmo productivo (Santagata, 2002).

Lo anterior, expone que el surgimiento de esta economía se acompaña por una expansión significativa en el número de trabajadores dotados de altos niveles de especialización respecto a funciones tales como pensamiento analítico, toma de decisiones, flujo de ideas, capacidad de interacción con otros, imaginación, conocimiento sustantivo y experiencia. A la luz de tales observaciones, tópicos como creatividad, innovación, capital humano, clase creativa y ciudades creativas son parte fundamental en las agendas de investigación relacionadas. Particularmente, la creatividad constituye un término cuyo significado es por ningún medio evidente en sí, sin embargo, infiere una clasificación sustantiva imperiosa ya que no es sólo un término ambiguo, sino que constituye un foco de atención prevaleciente para asuntos relacionados con esquemas de crecimiento y desarrollo (Scott, 2010).

$\mathrm{Al}$ respecto, la base teórica inicial que vincula estas tendencias con el crecimiento económico, parte de los enfoques neoclásicos de Solow (1956) y Swan (1956). El modelo de Solow explica cuáles son las fuentes de crecimiento de la producción en economías capitalistas al incorporar los supuestos habituales del análisis clásico con factores exógenos. Posteriormente, en la mitad de la década de los ochenta, un grupo de teóricos, 
entre ellos Romer (1986), Lucas (1988), Rebelo (1991), Grossman y Helpman (1990) muestran su insatisfacción por los modelos exógenos, postulando tendencias con determinantes a largo plazo. Empero, el modelo parteaguas es el de Romer (1986), cuya característica distintiva es que introduce una función de producción con externalidades de capital. Se sugiere que, las externalidades positivas pueden tener un impacto en todas las empresas de la economía debido al aprendizaje por la práctica y el desbordamiento del conocimiento.

No obstante tales presunciones, Lucas (2003), Barro y Sala-i-Martin (2004) manifestaron interés en la renovación de la teoría endógena, por lo que condujeron a modelos donde las economías registran una tasa de crecimiento constante, lo cual evita llegar al estado estacionario. Así pues, se concentran esfuerzos hacia la determinación de factores compuestos de la intensificación de capital humano, especialización y utilización de la tecnología como factor interno, donde los rendimientos crecientes generan innovación.

Al respecto, Sala-i-Martin (1990) elabora una importante contribución al entendimiento de la interminable cuestión acerca del crecimiento económico. La función de producción debe mostrar rendimientos constantes en los insumos que pueden ser acumulados. Entonces, postula dos formas; la primera, de acuerdo con Alfred Marshall (1890) es introducir los rendimientos crecientes a escala a nivel agregado y, los rendimientos constantes, a escala a nivel de firma, lo que puede ser formulado a través de externalidades de la producción o spillovers. La segunda forma, es el llamado enfoque Chamberliano para los rendimientos crecientes. Existen rentas que pueden ser asignadas a actividades no directamente productivas, pero que pueden contribuir a la expansión de la frontera de conocimiento, tal como $R \& D$ (Sala-i-Martin, 1990). Según Barro y Sala-i-Martin (2004), $R \& D$ es una importante fuente de crecimiento y son razonadas como nuevas variedades de bienes de capital.

De esta manera, la propuesta parte de supuestos instalados en la percepción de que la utilización y organización del potencial humano es clave, ya que disminuye la incertidumbre al trasladar el conocimiento, fuente inagotable capaz de multiplicar los rendimientos de capital, favorecer la innovación y dotarse de factores internos-externos.

Particularmente, la cultura es vista como una especie de esfera privilegiada de la creatividad, libre de condiciones físicas, lo cual implica innovación, es decir, el dominio de la ciencia y tecnología donde en algunas ocasiones la imaginación humana es finalmente libre en la búsqueda de inimaginables formas de autoexpresión (Scott, 2010).

Tal afirmación supone que la economía cultural emerge del contexto endógeno relacionado con la propuesta de Romer (1986) y Sala-i- 
Martin (1990), dado que el componente creativo impone la necesidad de articular el crecimiento alrededor de factores internos. Entonces, la valoración acerca de las variables atribuibles al crecimiento económico desde las vertientes local y regional, cambia. Ante tal esquema, se puede distinguir tres términos íntimamente relacionados: aprendizaje, creatividad e innovación.

El aprendizaje para (Scott, 2010) provee importante fundamentos de información y procedimientos para la actividad creativa; creatividad en sí se relaciona con pensamiento y acción, dirigido a la producción de puntos de vista y percepciones que pueden o no eventualmente tener significado tangible; innovación deriva de estos puntos de vista y percepciones, pero se dirige específicamente a su implementación en el dominio diverso de la aplicación práctica. Paralelamente, Scott (2010: 119-120), señala que:

1) El pensamiento creativo es siempre moldeado por el conocimiento y las habilidades de los individuos, a través de la educación y socialización en un contexto histórico y geográfico definido. 2) Los productos del trabajo creativo deben ser reconocibles como tales por otros. 3) Las estructuras de las transacciones externas dentro de las cuales las firmas y trabajadores operan tienen substanciales efectos para el desencadenamiento de la mano de obra creativa. 4) El ámbito de la creatividad sugiere que sus actividades son propensas a ser marcadas por patrones de dependencia, regulados por amplias condiciones sociales.

En este tenor, la regulación entre la actividad productiva y otros factores clave aumentan el debate acerca de las implicaciones de la creatividad y cultura, hacia horizontes donde se perciben cada vez más como catalizadores del crecimiento económico.

\section{Geografía económica creativa y acercamiento a la lógica espacial de industrias culturales}

La intensificación de la relación entre industrias altamente creativas, traslada la atención al contexto territorial, en relación con el rol fundamental de las formas de clusterización (Lazzeretti et al., 2008; Mommas, 2004 y Santagata, 2002). El rol estratégico de los recursos culturales y las dinámicas de aglomeración evidencian la habilidad de activar nuevas cadenas productivas y revitalizar ciudades y regiones.

$\mathrm{Al}$ respecto, se considera que los postulados de la nueva geografía económica constituyen el primer espacio, desde el cual, la tendencia de tales industrias marca su composición territorial geográfica. El imperativo del sistema de producción y los procesos de aglomeración parten de 
teorías conceptuales con determinantes de crecimiento local, donde los factores internos se utilizan intensamente.

La nueva geografía económica enfoca su atención en la espacialización de los factores endógenos y las formas de organización creativas. Particularmente Fujita y Thisse (2007) postulan que el crecimiento obedece a una lógica de causación circular en la que los encadenamientos hacia atrás y hacia delante de las empresas conducen a una aglomeración de actividades que se autorefuerzan progresivamente.

En la misma vertiente, Storper y Walker (1989) proponen la dinámica espacial del crecimiento industrial. La hipótesis principal es que las industrias originan regiones como sitios de actividad económica. Los patrones básicos de localización industrial y crecimiento regional pueden ser causados por procesos endógenos de la industrialización capitalista, más que por variantes exógenas, lugares, recursos o consumidores.

Las industrias de rápido surgimiento se ven unidas a las capacidades locacionales para alcanzar ganancias normales, lo cual permite atraer recursos y mano de obra. La productividad se incrementa, atraída por la división y la integración del trabajo, disminuyendo precios y aumentando la concentración espacial. Los rendimientos crecientes son producto de las economías internas, constituidas por la concentración, intensificación y división social del trabajo (Storper, 2005). Se intuye que el surgimiento de nuevas industrias en la sinergia espacial creativa responde a estos postulados, donde la dotación de factores de producción dependen, en gran media, de la utilización endógena de variables, principalmente el capital humano.

Las industrias de rápido surgimiento se ven unidas a las capacidades locacionales para alcanzar ganancias normales, lo cual permite atraer recursos y mano de obra. La productividad se incrementa, atraída por la división y la integración del trabajo, disminuyendo precios y aumentando la concentración espacial. Los rendimientos crecientes son producto de las economías internas, constituidas por la concentración, intensificación y división social del trabajo (Storper, 2005). Se intuye así, que el surgimiento de nuevas industrias en la sinergia espacial creativa, responde a estos postulados, donde la dotación de factores de producción dependen en gran media de la utilización de variables endógenas, principalmente el capital humano.

Desde esta perspectiva, los cambios en los análisis vertidos a la dinámica económica en el espacio son adaptativos y cambiantes. Así, un nuevo paradigma llamado variedad relacionada (Frenken et al., 2007; Boschma y Iammarino, 2007), surge de los estudios de la geografía económica evolutiva. 
Boschma y Iamarino (2007) encuentran que demasiada confianza en las fuentes del conocimiento local puede ser perjudicial para el aprendizaje interactivo y la innovación; de manera tal que cuando las empresas locales se orientan hacia adentro su capacidad de aprendizaje puede debilitarse, a medida que pierden su talento para la innovación y son incapaces de responder a nuevos desarrollos. Tal tipo de bloqueo puede ser resuelto o evitado mediante el establecimiento de redes extralocales.

Conectarse a tales redes es considerado como condición previa para la sobrevivencia de las firmas de un cluster. Estos vínculos extraregionales atraen nuevo conocimiento a través de una amplia gama de sectores localizados, la condición es que estos flujos de conocimiento estén relacionados a la especialización sectorial, si el conocimiento externo no está relacionado a la base industrial, entonces no podrá ser asimilado (Boschma y Iammarino, 2007). Es decir, la mayor cantidad de innovaciones ocurren a través de spillovers entre sectores, más que dentro del mismo sector, siempre y cuando éstos estén relacionados y compartan competencias. Como tal, el concepto va más allá de la dicotomía tradicional de economías de localización y externalidades y es utilizado recientemente en industrias de gran contenido creativo, particularmente a través del proceso activo de relaciones cognitivas entre diferentes industrias.

La vertiente de análisis evolutiva indica mecanismos para estimular nuevos patrones de crecimiento en variados contextos territoriales e institucionales. En esta propuesta, Dawley (2013) afirma que actualmente el surgimiento de una nueva industria puede ser habilitado o restringido por ambientes y contextos condicionados por características y competencias inherentes a trayectorias locales previas. Esto se refiere al reciente progreso para entender los mecanismos que estimulan nuevos patrones de crecimiento, al desarrollar la integración del análisis a nivel firma, en un amplio y territorialmente variado contexto institucional, para conectarse a una amplia gama de actores y contextos multiescala.

Trayectoria de dependencia es un concepto que se aplica a las industrias culturales para sugerir que el estado presente de condiciones socioeconómicas y crecimiento económicocultural en cualquier lugar es altamente dependiente de decisiones, localidades y dinámicas que previamente afectan al lugar (Unesco, 2013).

La creación de trayectorias es un elemento latente para las de dependencia, condicionadas por un conjunto de características locales, tecnológicas e industriales y factores dependientes del lugar, lo que ayuda a explicar porqué trayectorias características han crecido en lugares particulares y no en otros (Dawley, 2013). Es así que, el desplazamiento de industrias culturales y sus dinámicas endógenas se relacionan a la geografía econó- 
mica y su variante evolutiva, lo cual extiende las cuestiones relativas al espacio y variables de crecimiento en regiones creativas.

\section{Cluster, distinción productiva y dinámica de industrias culturales}

Las formas de la economía cultural en el espacio territorial, así como las dinámicas de organización de la producción tienden a materializarse en forma de densas aglomeraciones. Esta propuesta se refiere a sinergias de innovación percibidas en clusters industriales; los que adquieren ventajas competitivas específicas del lugar, por razón de las simbologías culturales locales congeladas en sus productos. Ello implica especial atención hacia los términos en que se vincula la lógica espacial, así como los parámetros y las formas de la concentración territorial que permiten la ejecución competitiva, por un lado, y su potencial creativo, por otro.

Por consiguiente, en tanto persista el aumento en su dinamismo, motivado por la demanda y el consumo, se deberá atender en mayor medida la dinámica industrial que se lleva a cabo en sectores selectos de sus productos y las formas en las cuales dicha economía se organiza en lugares específicos.

Así, se tiene que los nuevos sectores se componen por procesos de producción relativamente desarticulados, y sus productos son atraídos principalmente hacia grandes áreas metropolitanas, algunas de las más avanzadas expresiones de estas tendencias se observan en grandes ciudades mundiales como Los Ángeles, Nueva York, Londres, París, Berlín, Roma y Tokio. La propensión de las firmas de los sectores de productos culturales es converger en clusters espaciales distintivos dentro de la ciudad, lo cual es reflejo de una estructura organizacional, en la que cada unidad de producción individual es atrapada en un amplio sistema de interacciones socioeconómicas, sobre las cuales depende su supervivencia. Las firmas tienen un enorme incentivo para permanecer juntas en comunidades dentro de la ciudad, ya que la proximidad mutua mejora considerablemente la disponibilidad de economías de aglomeración y los efectos de rendimientos crecientes (Scott, 2004).

Santagata (2002) afirma que su conformación territorial productiva se sustenta en el capital cultural tangible e intangible, el cual es el soporte de la producción de bienes materiales e inmateriales a través de dos insumos estratégicos: creatividad humana y actividad intelectual.

Así, los clusters industriales-culturales se convierten en un buen ejemplo del crecimiento endógeno sustentable; se promueve un patrón de crecimiento a través del desarrollo de pequeñas y medianas firmas las cuales se encuentran intensamente integradas dentro de un territorio y en la comunidad local. 
Una de las características más significativas es la interdependencia de sus firmas. La atmósfera industrial y el contacto favorecen el intercambio de bienes especializados, donde las continúas y repetidas transacciones promueven la circulación de la información. Hacia el interior de las aglomeraciones, Santagata (2002) aprecia la presencia de contratistas que verifican la calidad de los bienes y servicios; también, que el conocimiento tácito, la confianza mutua y la acumulación de capital social son producto de los tratos permanentes entre la sociedad local y cultura.

Es así que, estas industrias se posicionan en la lógica del crecimiento, los clusters son la expresión particular de la economía cultural, adaptación espacial geográfica con sistemas de producción y capital humano en constante dinamismo creativo, lo que a su vez transforma los insumos en bienes diferenciados y servicios especializados de demanda global.

Así, los flujos de combinaciones productivas surgen y se expanden sobre ventajas competitivas específicas de diversos clusters creativos. La intensa apertura del comercio global hace posible el establecimiento de ventajas competitivas permanentes y capaces de atacar nuevos mercados. Esto, se representa por industrias como la producción de programas de televisión, música, juegos electrónicos, turismo, arquitectura, publicidad, diseño de modas, joyería, entre otros, las cuales representan de $4 \%$ a $8 \%$ del total del porcentaje de empleo actualmente (UNESCO, 2009).

De tal manera que no solamente se observa una multiplicidad de centros de producción activos, sino se espera el surgimiento y desarrollo de otros, junto a la expansión de un gran sistema de localidades de producción satélite, un ejemplo de esto, es la existencia de un conjunto diverso de pequeñas áreas metropolitanas con economías culturales florecientes como los centros vacacionales y de turismo como Miami y San Diego; Centros de convenciones y resorts como Orlando y Las Vegas; aglomeraciones de música como Austin, y Nashville; lugares artesanales y de herencia cultural como Santa Fe, Nuevo México y Savanah, lugares que mantienen altos niveles de servicios culturales y son atractivos para vivir y trabajar (Scott, 2010).

En los territorios donde las ventajas locales apropiadas se encuentren disponibles es posible analizar el contexto de industrias florecientes, a través de la caracterización del sistema de producción interno, su componente creativo, la relación en el espacio territorial y principales variables motrices. Para tales efectos se propone la construcción de un modelo en el que la economía cultural depende de dos factores: capital humano (creatividad) y sus dinámicas en el espacio geográfico (dinámicas de aglomeración). 


\section{Modelo de crecimiento e innovación para cluster creativo de industrias culturales. Teoría y aplicación}

En esta sección se realiza la aplicación del método analítico-teórico planteado previamente. Se pretende proveer una herramienta de aplicación funcional, siguiendo un acercamiento multidisciplinario que integra parámetros de la economía cultural, teoría de crecimiento endógeno y geografía económica. El análisis permite la identificación de los patrones de crecimiento económico en clusters creativos de industrias culturales florecientes, al utilizar el sistema de producción local y modelado econométrico de los determinantes económicos-creativos en un espacio geográfico definido.

Las perspectivas consideradas parten de (Lazzeretti et al., 2009), quienes generalizan una ecuación lineal a formas más flexibles al disminuir restricciones en las elasticidades, lo que permite incluir factores alternos, de manera que las variables se dividen en distintos conjuntos en la misma operación. Particularmente, el modelo propuesto considera los siguientes acercamientos:

Productividad creativa localizada -en base al LQ (Location Quotient) de empleo-; Economías de aglomeración y organización de la industria; Capital humano $(R \& D)$; Especialización (rendimientos crecientes, externalidades acumuladas, innovaciones en términos de patentes); Consumo; Variedad relacionada (Boschma y Iammarino, 2007) diversifica el perfil creativo de un sistema de producción local.

A partir de análisis vertidos, se considera utilizar el cociente de localización (LQ) para verificar la motricidad de la industria cultural en un lugar determinado, lo que permite comparar su especialización en relación al promedio nacional (Lazzeretti et al., 2008), entonces se puede elaborar un estimado empírico de cada industria y su participación en la economía general a través del empleo.

Se desagrega el procedimiento que permite incorporar los elementos necesarios para poder: a) Estimar el crecimiento económico local con base en la industria creativa del cluster; b) Evaluar la concentración del cluster creativo respecto a la organización de la industria y economías de aglomeración, a través de las variables de capital humano, especialización $(R \& D)$, consumo y variedad relacionada; $c$ ) Determinar la correlación entre la producción económico-creativa (prácticas registradas, patentes, inversión) y rendimientos crecientes del cluster en el sustento de la economía regional.

La propuesta que se generalizará parte de la variable dependiente (cluster creativo) y variables independientes (Economías de aglomeración, 
especialización, capital humano, innovación, empleo y consumo). Entonces:

$$
\gamma c=\alpha X_{1}^{\beta 1}, X_{2}^{\beta 2}, X_{3}^{\beta 3}, X_{4}^{\beta 4}, X_{5}^{\beta 5}
$$

Donde: $\gamma c$ es el LQ de empleo en industrias culturales de gran contenido creativo de un cluster, $\mathrm{X}_{1}$ a $\mathrm{X}_{5}$ es el conjunto de variables que representan a las economías de aglomeración, el capital humano, especialización, consumo y variedad relacionada, en tanto $\alpha, \beta_{1}$ a $\beta_{5}$ son el conjunto de parámetros; $\gamma$ c significa que existe motricidad en la industria y empleo creativo.

La propensión de las firmas de los sectores de productos culturales es converger en clusters espaciales distintivos como reflejo de una estructura organizacional, en la cual, cada unidad de producción individual es atrapada en un amplio sistema de interacciones socioeconómicas de las que depende su supervivencia. Las firmas tienen un enorme incentivo para permanecer agrupadas, ya que la proximidad mutua mejora considerablemente la disponibilidad de economías de aglomeración y los efectos de rendimientos crecientes. Las firmas de las industrias de los productos culturales con estas características son sujeto de presiones competitivas y organizacionales, tales como la aglomeración en densos clusters o distritos industriales (Power y Scott, 2004).

La función (ecuación 1) puede alinearse usando logaritmos, para producir una ecuación lineal larga:

$$
\operatorname{In}(\gamma)=\operatorname{In}(\alpha)+\beta_{1} \operatorname{In}\left(X_{1}\right)+\beta_{2} \operatorname{In}\left(X_{2}\right)+\beta_{3} \operatorname{In}\left(X_{3}\right)+\beta_{4} \operatorname{In}\left(X_{4}\right)+\beta_{5} \operatorname{In}\left(X_{5}\right)
$$

A partir de esta lógica, adicionalmente la ecuación 2 se puede generalizar a formas más flexibles, siguiendo la función translog (Berndt y Christensen, 1973, citado en Lazzeretti et al., 2009), la cual relaja las restricciones sobre elasticidades al substituir factores que permiten un segundo orden de efectos cruzados:

$$
\operatorname{In}(\gamma c)=\sum_{\mathrm{j}=\mathrm{i}} \operatorname{In}\left(\mathrm{T}_{\mathrm{ij}}+\mathrm{Q}\right)+\operatorname{In}\left(E_{\mathrm{j}}\right)^{(\beta+\varphi-1)}+\operatorname{In}\left(K_{\mathrm{j}}\right)^{(\beta+\varphi-1)}+\sum_{g=1}^{G} P_{g, j} H_{g, j}+\operatorname{In}\left(C_{j}\right)
$$

De acuerdo con el marco teórico, las variables explicativas se dividen en cinco grupos:

1. Economías de aglomeración del sistema de producción local (representado por $j$ en el modelo), las cuales se constituyen por varios indicadores, siguiendo a Storper y Walker (1989) y las formas marshallianas mencionadas por Scott (2010) parten de la industrialización geográfica e 
incluyen la estructura y organización de la industria, mercado laboral local y proveedores especializados.

Es importante señalar que, el tamaño promedio de la firma en el sistema de producción local tiene preferencia por la organización de la industria en pequeñas y medianas empresas, entonces:

$$
\text { Tamaño de firma } a_{j}=\frac{T_{j}}{F_{j}}
$$

Donde $T$ es el empleo (empleos) y $F$ es el número de firmas. Un segundo indicador, propuesto por Lazzeretti et al. (2009), controla el efecto del tamaño de la firma en industrias de gran contenido creativo en el sistema de producción local:

$$
\text { Tamaño de firma }{ }_{j}=\frac{T_{i j}}{F_{i j}}
$$

Donde $j$ es el sistema de producción local e $i$ es la industria de alto contenido creativo. Por su parte, el mercado de trabajo local calificado se estima a través del número de trabajadores calificados en industrias culturales:

$$
Q_{i j}=\frac{Q T_{i j}}{T_{i j}}
$$

Donde $Q$ es el empleo calificado, lo que conlleva a la variable de especialización representada por $(E)$.

2. Los modelos de crecimiento endógeno utilizan a la especialización (E) como fuente del crecimiento. La idea de que esta variable lleva a retornos crecientes a escala se formaliza en Ethier (1982). La función de producción es creciente del número de insumos intermedios utilizados en la producción, así, mientras mayor sea el número de insumos intermedios utilizados, mayor es el producto final.

En el modelo propuesto, al estimar el crecimiento endógeno de un cluster a partir de $(E)$, se encuentra con la intensificación de los rendimientos crecientes y externalidades acumuladas $\left(\beta+\varphi^{-1}\right)$, esto es el sector de bienes intermediarios, el cual utiliza el diseño del sector de la investigación $(R \& D)$, junto al capital para producir un gran número de bienes duraderos, los cuales están disponibles para el uso de la producción de bienes finales en cualquier momento.

La investigación y el desarrollo $(R \& D)$ crean diseños para nuevos tipos de bienes de capital, donde los spillovers $\left(\beta+\varphi^{-1}\right)$ del conocimiento son fundamentales, incrementándolos es la forma en que la integración acelera el crecimiento. En la propuesta de Romer (1989) esto quiere decir que existen rendimientos crecientes a escala en la producción de función, 
lo cual provoca que la integración tenga un efecto de crecimiento dirigido por el conocimiento.

Por otro lado, para Sala-i-Martin (1990) en el crecimiento endógeno se concentran los esfuerzos hacia la determinación de factores compuestos de la potencialización de capital humano, la especialización y la determinación de la tecnología como factor interno, donde los rendimientos crecientes tienen que generar innovación. Asimismo, añade a la teoría del crecimiento el acercamiento necesario para trascender al estado empírico del conocimiento. En su teoría de crecimiento endógeno, Sala-i-Martin (1990) propone introducir los rendimientos crecientes a escala a nivel agregado y los rendimientos constantes a escala a nivel de firma. Esto puede ser formulado a través de externalidades de la producción o spillovers; la decisión de cada firma afecta la producción de otras firmas, esto bajo algunas condiciones genera crecimiento endógeno. Esto es:

$$
Y_{t}=A K^{\beta}{ }_{t} L_{t}^{1-\beta} k_{t}^{\varphi}
$$

Donde:

$Y=$ denota el producto

$A=$ un nivel de tecnología dado, que se supone constante

$K_{\mathrm{t}=}$ capital privado

$k_{\mathrm{t}=}$ capital agregado en la economía

$L_{\mathrm{t}=}$ fuerza de trabajo

$\beta=$ rendimientos crecientes

$\varphi=$ externalidades (spillovers)

Las firmas individuales no piensan que afectan $k$, así que lo toman como algo dado. En forma agregada, el total del capital igualará la suma del capital individual, es decir, $k-K$; entonces, la función de producción agregada será:

$$
Y_{t}=A K^{\beta} L_{t}^{1-\beta}
$$

Se debe señalar que si la externalidad es "correcta" $\left(\beta+\varphi^{1}\right)$ se obtendrán rendimientos constantes al capital y rendimientos crecientes a escala.

El que existan retornos crecientes no es una condición suficiente para que haya crecimiento endógeno de largo plazo positivo. Se requieren al menos retornos constantes a los factores que se pueden acumular. En otras palabras, en el modelo de rendimientos crecientes a escala $(a+\beta=1)$ teniendo crecimiento de estado estacionario $\left(r_{\mathrm{k}}>0\right)$, la función de producción debe exhibir rendimientos crecientes de los insumos que pueden ser acumulados, $\beta-1$. 
$\mathrm{Al}$ respecto, se puede definir a un cluster de industrias culturales florecientes como un escenario donde la endogenización de factores de producción, como especialización y la tecnología $(R \& D)$, propician que procesos innovadores como los de la economía cultural se aglomeren y especialicen a través de $\left(\beta+\phi^{-1}\right)$.

Paralelamente $(E)$ y $(R \& D)$ representan, hipotéticamente, una mayor estabilidad del crecimiento económico regional, sustentado en innovaciones que a la vez representan el número de registro de patentes, prácticas registradas, propiedad intelectual. Esto es la densidad de patentes locales:

$$
E_{R \& D}=P \alpha_{t j} / T_{j}\left(\beta+\phi^{-1}\right)
$$

Las patentes locales representan el número de innovaciones registradas por los trabajadores creativos de un cluster cultural, éstas surgen de la intensificación de las externalidades acumuladas, beneficios propios y únicos de cada cluster. $\left(\beta+\phi^{-1}\right)$ aparece como factor representativo de esta interacción en el SPL, se miden al final de la ecuación en función de la variedad relacionada.

3. Por otro lado, $K$ es una variable muy importante en la construcción del modelo. Su inclusión se sustenta en las teorías de crecimiento endógeno, especialmente las de Romer (1989) y Sala-i-Martin (1990). La característica distintiva del modelo del primero es que introduce una función de producción con externalidades del capital. Se sugiere que las externalidades positivas pueden tener un impacto en todas las empresas de la economía debido al aprendizaje por la práctica (learning by doing) y el desbordamiento de los conocimientos (knowledge spillovers).

Con el objeto de generar crecimiento endógeno asume retornos crecientes a escala, pero con economías externas a la firma, de modo de asegurarse la existencia del equilibrio competitivo. La función de producción asumida es:

$$
Y_{i}=F\left(k_{i}, L_{i}, k\right)=K_{i}{ }^{\beta} L_{i}^{(1-\beta)} K^{n}
$$

Donde:

$Y_{i}=$ Producto de la firma $i$

$k_{i}=$ Stock de capital firma $i$

$L_{i}=$ Empleo firma $i$

$K_{i}=$ Stock de conocimiento

$i=1$ 
Se asume "N" firmas con un "N" suficientemente "grande" y que todas las firmas son idénticas. Así: $k=\mathrm{NK}_{\mathrm{i}}$. Dado que "N" es grande, cada firma asume que el stock agregado de capital o stock de conocimiento está dado y ella no lo puede afectar. En este sentido, los retornos crecientes son externos a la firma. Sin embargo, es claro que si todas las firmas incrementan su $K_{\text {i., }}$ el stock total del conocimiento aumentaría, beneficiándose todas. Debido a que hay retornos crecientes a escala en función de producción, la acumulación de capital puede generar crecimiento endógeno (Romer, 1989).

De esta manera, la acumulación de capital humano $(K)$ determina que el nivel de crecimiento, el cambio tecnológico y la innovación se encuentran en el corazón del crecimiento económico; el conocimiento se traslada dentro de bienes con valor práctico (Romer, 1989). En esta teoría, la potencialización del capital humano intensivo se expone como la clave del crecimiento económico.

En el modelo $K$ se estima a través del número de personas formalmente educadas (graduados de universidades y posgraduados), quienes participan en el sistema de producción local. Esto, estimando el equivalente insertado en el SPL.

$$
\text { Capital Humano }(K)_{j}=T_{j} \sum T_{S F E j}^{(\beta+\varphi-1)}
$$

Donde $T_{j}$ representa el trabajo total del spL y $T_{S F E j}$ el número de trabajadores en el sistema formalmente educado. El sector de la investigación utiliza al capital humano $(K)$ y el stock (acumulación) de conocimiento existente, para producir nuevo conocimiento, en el modelo propuesto esto se muestra en $\left(\beta+\phi^{-1}\right)$ ya que son factores intermedios basados en la transmisión de ideas que propician la productividad constante, estimados al final de la ecuación a través de la variable variedad relacionada.

4. Lo anterior propone entonces mantener una constante, lo que se asume en los argumentos recientes de Boschma y Iammarino (2007), quienes formulan una extensión a la propuesta alrededor de las economías de localización, especialización sectorial y la diversificación de la economía regional sustentada en economías externas, junto al desparramamiento del conocimiento. Encuentran que, la excedida confianza en las fuentes del conocimiento local puede ser perjudicial para el aprendizaje interactivo y la innovación. El conocimiento se propaga con eficacia entre sectores cuando las complementariedades existentes entre éstos se comparten en términos de competencias.

Tales complementariedades existen en términos de variedad relacionada, misma que se identifica como sectores industriales que se vinculan 
en términos de competencias complementarias o intercambiables (cognitivamente).

Así, la variedad relacionada en el modelo, se estima usando en índice de nivel entrópico de tres dígitos propuesto por Boschma e Iammarino (2007). El valor del indicador entrópico incrementa el perfil creativo más diverso de un sistema de producción local:

$$
\text { Variedad Relacionada } a_{j}=\sum_{g=1}^{G} P_{g, j} H_{g, j}
$$

Donde:

$$
P_{g, j}=\sum_{i e S g} p_{i, j}
$$

es la suma de sectores de dos y tres dígitos de cada industria a la participación total del empleo,

$$
P_{i, L P S}=L_{i, j} / \sum_{i=1} L_{i, j}, \mathrm{y} H_{g j}=\sum_{i e S g} \frac{p_{i, j}}{P_{g, j}} \log _{2}\left(\frac{1}{p_{i, j} / P_{g, j}}\right)
$$

La propuesta no niega la existencia ni el beneficio de las economías externas en un lugar, añade a tal análisis la posibilidad de mantener en equilibrio el sistema innovador local, al inducir variables alternativas externas a través del conocimiento, ejemplo de esto, es el flujo migratorio. Su medición atañe importancia a la forma empírica de obtener resultados respecto a las externalidades o spillovers de la industria, factor presente en la variable de especialización $(E)$ y capital humano $(K)$.

5. Otra de las variables incluidas es el consumo (C); Throsby (2001) señala que la noción de cultura como economía y su interpretación como algo incluido en un medio económico nos lleva a la propuesta de que su producción y consumo se pueden situar dentro de un marco industrial. Los bienes y servicios culturales producidos y consumidos se pueden considerar como mercancías, en los mismos términos que otras producidas en el sistema económico.

Para Scott (2008) el consumo y la mercantilización de la cultura provocan desafíos que debilitan la estructura económica convencional de los gobiernos locales, quienes tienen que renovar las formas de organización en el espacio regional, político y social. En el modelo, el consumo se especifica: 


\section{Consumo $=$ PIB Región/Pat}

A partir de lo anterior, la variable $(C)$ se identifica con el proceso de producción, consumo e intercambio de la creatividad de un sistema de producción localizado. Pat representa el índice de participación de un cluster creativo en el PIB del sistema de producción local, por ello puede utilizarse en términos de la variable más representativa, siempre y cuando ésta se mida por unidades (número de patentes, prácticas, registros, permisos).

En consecuencia $(C)$ en el modelo permite estimar el consumo y la demanda final de una industria, esta variable puede emplearse como una proxy asociada al crecimiento económico regional.

\subsection{Alcances e implementación de resultados}

El modelo considera responder a la hipótesis general vertida en torno a la utilización de industrias culturales como factor propulsor de la economía, la cual retoma la cuestión acerca de la ventaja de estas complejas tendencias para los propósitos del desarrollo económico.

En contexto particular, la propuesta se dirige al estudio de clusters creativos florecientes, donde se advierte la posibilidad de ventajas competitivas al identificar la actividad creativa, midiendo el grado de concentración y motricidad de la industria o actividad localizada con base en el LQ (Location Quotient), herramienta que conjetura la relativa especialización del lugar y la industria respecto al promedio nacional.

De forma práctica, se determinan los LQ o cocientes de localización, utilizando y dividiendo los factores de la industria, en términos del número de empleados creativos y el total nacional en la misma (ver ecuación $3)$. Así, $(\gamma c)$ representa el índice de concentración regional creativo medido a partir del coeficiente LQ.

Por su parte, las economías de aglomeración del sistema de producción local ( $j$, en el modelo) tienen representatividad empíricamente medible a través de indicadores específicos, en el caso particular representan la organización de la industria y mercado laboral. Su apreciación en la ecuación principal $\Sigma_{\mathrm{j}=1} 1 n\left(\mathrm{~T}_{\mathrm{ij}}+\mathrm{Q}\right)$ contribuye a identificar en un cluster, el tamaño de las firmas en el sistema de producción local $(j)$, al dividir el empleo $(T)$ entre el número de estas $(F)$. Los resultados exponen la estructura interna del cluster y las empresas relacionadas a la actividad creativa, lo que asume marginalidad productiva relacionada a la ganancia y rentabilidad de la industria en crecimiento.

En tal apreciación, el efecto del tamaño de la firma indica que están constituidas en términos organizacionales como redes complementarias 
especializadas, que trabajan en competencia y colaboración con otras. La naturaleza creativa de la producción incentiva una mayor desintegración, ya que los productores buscan constantemente nuevas combinaciones de mano de obra con la finalidad de permanecer en el juego del mercado a través de innovaciones y la diferenciación del producto.

$\mathrm{Al}$ respecto, la variable $(Q)$ evalúa al mercado de mano de obra local calificado, el cual, de forma práctica, se promedia al dividir el número de trabajadores calificados en industrias culturales $\left(Q T_{i j}\right)$ entre los trabajadores en el sistema de producción local de alto contenido creativo $\left(T_{i j}\right)$. Estas variables permiten esgrimir una de las constantes medulares de estas industrias como factor propulsor del crecimiento económico, el empleo.

La estimación de este mercado indica la dinámica del capital humano y su contribución marginal a la producción del cluster; la mano de obra local se compone por diferentes habilidades, aptitudes y sensibilidades, reflejo del rango de demanda de este capital y el empleo en el sistema. En consecuencia, se promueve la presencia de capacidades distintivas entre firmas y trabajadores, interconectando capacidades, lo que incentiva la convergencia de productores en la localidad y la existencia de recursos de reforzamiento interno al incrementar las ganancias o economías de aglomeración.

Para efectos funcionales, la calidad del mercado de trabajo local se propone como herramienta de estimación del potencial de crecimiento en una industria floreciente. Si ésta detenta una base de mano de obra calificada en ( $j$ ), entonces, puede promover las competencias básicas para considerar la utilización y especialización del capital humano existente, en virtud de innovaciones que finalmente aumenten la productividad.

Lo anterior extiende la medición de variables a la especialización $(E)$, la ecuación principal (ver ecuación 3) indica su comportamiento. La utilidad de $(E)$ parte de asunciones endógenas, las cuales la destacan como fuente de crecimiento. Este enfoque, determina que capital humano, especialización y tecnología, como factores internos, potencializan el crecimiento a largo plazo, al aumentar los rendimientos crecientes cuando se genera innovación. La respuesta a la dinámica creativa de industrias culturales y su utilización como factor propulsor de la economía se sostiene en la explotación de tales variables.

En la propuesta $(E)$ el factor $\left(\beta+\phi^{-1}\right)$ es clave y ejemplifica la presencia de rendimientos crecientes y externalidades acumuladas, es decir, el sector activo de bienes intermediarios que a la vez utiliza al sector de la investigación $(R \& D)$. La función principal es incentivar la creación de innovaciones, lo que mantiene la estabilidad y crecimiento sostenido en un cluster floreciente, éstas se estiman a través del número de patentes, prácticas registradas y propiedad intelectual. 
En el modelo, la densidad de patentes locales están en función de $E_{R \& D}$ y son producto de la división entre éstas y el número de trabajadores en el sistema de producción local $P a_{t j} / T_{j}$. Los spillovers se suman a la ecuación como factor de cambio indispensable, esto acelera los procesos internos característicos de cluster creativo; lo que genera una cadena productiva donde la utilización efectiva del capital humano promueve la existencia del flujo de conocimiento, de información y comunicación. Entonces, las industrias culturales como factor motriz de la economía son función de la creatividad, de la cual el producto final es la innovación.

Particularmente, la propuesta de la investigación advierte que los parámetros de crecimiento son relativos al número registrado de productos o servicios en una aglomeración. La creatividad es un bien imperceptible, no así, las innovaciones, producto de ellas. La determinación de la productividad marginal y los rendimientos crecientes a largo plazo son índice estimable de las posibilidades de una industria en la participación de la economía regional o local. Además, el registro de patentes, prácticas o propiedad intelectual reflejan la tendencia interna del trabajo y la explotación del capital humano, de no ser así, la utilidad en la ganancia sería nula. Es conveniente utilizar tal ecuación si se desea analizar las alternativas reales de la industria, el panorama final contribuirá al reforzamiento de variables poco incentivadas.

En esta vertiente se propone la estimación del capital humano. La acumulación de $(K)$ es susceptible a la intensificación, lo que lo convierte en un bien irreproducible y único, capaz de determinar el nivel de crecimiento junto al cambio tecnológico y la innovación real explotar el flujo de conocimiento efectivo. Enfatizar la acumulación de capital humano especializado mediante la inversión en educación formal y a través del learning-by-doing, asume que la creación del conocimiento se relaciona directamente con la inversión productiva, ya que una empresa que incrementa las competencias laborales simultáneamente produce mayor eficiencia.

La idea clave es que la inversión en educación, investigación y desarrollo es la fuente del crecimiento de largo plazo. En la propuesta se estima el número de personas formalmente educadas (graduados y posgraduados de universidades), participantes en el sistema de producción local. $(K)$ se vincula estrechamente a la variable $(E)$, como se observa en la ecuación 3 , ambas introducen externalidades $\left(\beta+\phi^{-1}\right)$, ambas se expresan de forma práctica al medir la variedad relacionada.

Finalmente, las imperfecciones de las variables del modelo prevén el bloqueo de los medios internos. En relación, Boschma (2007) encuentra que la excesiva confianza en las fuentes de conocimiento local puede tener 
implicaciones negativas, es decir, un sistema estacionario cerrado, donde el flujo no se renueva y evita la atracción de nuevas variedades.

Las complementariedades existen entre sectores en términos de variedad relacionada, que son sectores industriales vinculados a través de competencias (cognitivas).

Se estima con base en un índice entrópico, el cual incrementa el perfil creativo más diverso en un spl al sumar los sectores de dos y tres dígitos de cada industria respecto al empleo. Este índice contribuye a organizar la parte cognitiva presente en la producción creativa, característica de aglomeraciones de industrias culturales, y que son formas de medición suaves que complementan el análisis vertido sobre el comportamiento y contribución de éstas en el crecimiento.

Por su parte $(C)$ es la variable distintiva de la dinámica económica actual de tales industrias. Se estima a través del consumo de los bienes y servicios producidos en la aglomeración. La productividad marginal está en función del PIB regional entre el número de patentes del SPL Pat; su aumento es representativo del incremento en los retornos crecientes, la inversión y diversificación de la base creativa. De manera práctica, $(C)$ cuenta con dos índices importantes: la exportación de innovaciones registradas y la demanda interna. Se suma como variante significativa para consolidar las estimaciones reales acerca de la utilización de las mencionadas industrias como factor de crecimiento.

\section{Conclusiones}

El advenimiento de la nueva economía cultural, así como la consecuente extensión de sus productos y servicios se manifiesta en el funcionamiento del sistema productivo mundial, particularmente, en industrias de alto contenido creativo, las cuales revelan preferencia hacia procesos distintivos sobre el lugar en que se desarrollan.

Estas tendencias transforman la adaptabilidad general de la cultura al consolidar su presencia dentro del cauce de la economía capitalista, mediante la incorporación de sus productos a las formas más ambiguas de mercantilización, al tiempo que responden a una demanda creciente especializada. Esta fusión legitima el surgimiento de industrias culturales y la reconfiguración de los mecanismos disponibles para el crecimiento económico.

Tales industrias se despliegan como sectores integrados orientados a cubrir la demanda-consumo de productos y servicios simbólicos; estructuralmente se sustentan en un sistema de producción en red con interacciones endógenas, diseño flexible del mercado laboral y de suministros; se extienden geográficamente en dinámicas de aglomeración espacial, y 
sus procesos catalíticos parten de su capacidad de innovación. El notable incremento en la red mundial de estas concentraciones intensifica la diferenciación de productos, servicios y espacios en pugna por acelerar las ventajas competitivas. A pesar de ello, las teorías que las postulan como pivote del crecimiento, carecen de instrumentos que compensen las exigencias de su valoración y participación económica.

De esa forma, se propone un modelo de industrias culturales sujeto de medición, que reúne la vertiente del crecimiento endógeno y la nueva geografía económica para sistematizar sus vectores, distinguir el componente estratégico de la creatividad y su sistema de producción geográfico en dos niveles (cluster y economías externas o complementariedades). Se fundamenta un esquema de variables clave y se logra delimitar los patrones de extensión geográfica como clusters creativos.

Asimismo, se determina que el modelo está en función de dinámicas endógenas condicionantes, sugeridas como la concentración de actividades altamente creativas, economías de aglomeración, capital humano, especialización, variedad relacionada y consumo. El objetivo central es aportar un instrumento que evalúa la causalidad del ciclo económico, en virtud de la correcta organización de medios existentes. El esquema es asequible para casos de industrias florecientes que adviertan la posibilidad de ventajas competitivas y reflejen la inicial concentración de una actividad creativa motriz.

Asimismo, permite cotejar la conjetura central acerca de la contribución de una industria cultural aglomerada en el crecimiento económico de una región, ya que valora la conformación de la dinámica endógena y mide la incidencia de las variables explicativas que determinarán la capacidad competitiva de un cluster creativo potencial.

El modelo incorpora formas funcionales y relaciones económicas hipotéticas que pueden ser sólidamente confrontadas. Es un esquema teórico que especifica correlaciones validables empíricamente, como alternativa al estudio de la economía del conocimiento en el espacio territorial y el crecimiento. Como método, genera valor estadístico a las variables a partir de su estimación y es reproducible en distintos espacios. Por ello, se descartó como objetivo prioritario la exposición de un caso único.

Finalmente, el modelo contribuye en la reestructuración de procesos y en la canalización de las fortalezas de la economía con formas complementarias de organización e intervención. La naturaleza del campo de implementación generará nuevos debates y líneas alternativas de ejecución. 


\section{Bibliografía}

Adorno, Theodore W. (1991), The culture industry: selected essays on mass culture, Routledge, London.

Barro, Robert y Xavier Sala-i-Martin (2004), Economic Growth, Massachusetts Institute of Technology, Massachusetts.

Boschma, Ron y Koen Frenken (2006), "Applications of evolutionary economic geography", working paper, No. 06-26, Danish Reserch Unit for Industrial Dynamics, pp. 6-26.

Boschma, Ron y Simona Iammarino (2007), "Related variety and regional growth in Italy", Science and Technology Policy Research, núm. 62, University of Sussex, Brighton, pp. 1-24.

Caves, E. Richard (2003), Creative industries: Contracts between arts and commerce, Harvard University Press, Cambridge.

Dawley, Stuart (2013), "Creating new paths? Offshore wind, policy activism, and peripheral region development", Economic Geography, 90 (1), Newcastle University, Newcastle upon Tyne, pp. 91-112.

Ethier, J. Wilfred (1982), "National and international returns to scale in the modern theory of international trade", American Economic Review, 72 (3), American Economic Association, Pittsburgh, pp. 389-405.

Frenken, Koen, Frank Van Oort y Thijs Verburg (2007), "Related variety, unrelated variety and regional economic growth", Regional Studies Asociation, 41 (5), Routlegde, New York, pp. 685-697.

Fujita, Masahisa y Jacques Francois Thisse (2007), Economics of agglomeration. Cities, industrial location and regional growth, Cambridge University Press, Cambridge.

Gertler, Meric y Kate Geddie, (2008), "Architectural knowledge: reflexive dynamics between place and design", paper presented at the annual metting of the Association American of Geographers, 18 de abril, San Francisco. 
Grossman, M. Gene y Elhanan Helpman (1990), "Trade, innovation and growth", The American Economic Review, 80 (2), American Economic Association, Pittsburgh, pp. 86-91.

Hesmondhalgh, David (2002), The cultural industries, Sage publications, London.

Kloosterman, Robert (2008), "Walls and bridges: knowledge spillover between 'superdutch' aarchitectural firms", Journal of Economic Geography, Oxford University Press, Oxford, pp. 1-19.

Kloosterman, Robert (2010), "Building a career. Labour practices and cluster reproduction in dutch architectural design", Regional Studies, 44 (7), Taylor and Francis, London, pp. 859-871.

Krugman, Paul (1991), "Increasing returns and economic geography", Journal Political Economic, 99 (3), University of Chicago, Chicago, pp. 483-499.

Lash, Scott y Jonh Urry (1994), Economies of signs and space, Sage Publications, London.

Lazzeretti, Luciana, Rafael Boix y Francesco Capone (2008), "Do creative industries cluster? Mapping creative local production systems in Italy and Spain”, Industry and Innovation, 15 (5), Taylor and Francis, London, pp. 549-567.

Lazzeretti, Luciana, Rafael Boix y Francesco Capone (2009), "Why do creative industries cluster? An analysis of the determinants of clustering of creative industries", working paper presentado en Summer Conference on Copenhagen Business School, 17-19 de junio, Frederiksberg.

Lucas, Robert (1988), "On the mechanics of economic development", Journal of Monetary Economics, 22 (1), Elsevier, North Holland, pp. 3-42.

Lucas, Robert (2003), "Macroeconomic priorities", American Economic Review, 93 (1), American Economic Association, Pittsburgh, pp. 1-14. 
Markusen, Ann (2006), "Defining the cultural economy: industry and occupational approaches", Economic Development Quarterly, 22 (1), Cleveland State University, Cleveland, pp. 24-45.

Marshall, Alfred (1890), Principles of economics, MacMillan, London.

Mommas, Hans (2004), "Cultural clusters and the post-industrial city: towards the remapping of urban cultural policy", Urban Studies, 41 (3), Sage journals, London, pp. 507-532.

OCDE (Organización para Cooperación y el Desarrollo Económico) (2009), "Portal Estadístico", <http://www.oecd.org/statsportal/0 ,3352,en_2825_293564_1_1_1_1_1,00.html>, 9 de diciembre de 2009.

Power, Dominic y Allen J. Scott (2004), Cultural industries and the production of culture, Routledge, New York.

Pratt, C. Andy (1997), "The cultural industry production system: a case study of employment change in Britain, 1984-91", Enviroment and Planning, 29 (11), The London School of Economic and Political Science, London, pp. 1953-1974.

Pratt, C. Andy (2008), "Creative cities: the cultural industries and the creative class", Geografiska Annaler: Series B, Human Geography, 90 (2), Wiley, Suecia, pp. 107-117.

Prince, Rusell (2014), "Consultants and the global assemblage of culture and creativity", Transactions of the Institute of British Geographers, 39 (1), Royal Geographical Society-Institute of British Geographers, London, pp. 90-101.

Rebelo, Sergio (1991), "Long-run policy analysis and long-run growth", Journal of Political Economy, 9 (3), The University of Chicago Press, Chicago, pp. 500-521.

Romer, Paul (1986), "Increasing returns and long-run growth", Journal of Political Economy, 94 (5), The University of Chicago Press, Chicago, pp. 1002-1037. 
Romer, Paul (1989), "Endogenous technological change”, Journal of Political Economy, 98 (5), The Univesity of Chicago Press, Chicago, pp. 71-102.

Sala-i-Martin, Xavier (1990), "Lecture notes on economic growth (I): introduction to the literature and neoclassical models", working paper, No. 3563, National Bureau of Economic Research, Cambridge, pp. 1-47.

Santagata, Walter (2002), "Cultural districts, property rights and sustainable economic growth", International Journal of Urban and Regional Research, 26 (1), Wiley, Hoboken, pp. 9-23.

Scott, J. Allen (2000), "The cultural economy of Paris", International Journal of Urban and Regional Research, 24 (3), Joint Editors and Blackwell Publishers, Oxford, pp. 567-582.

Scott, J. Allen (2001), "Capitalism, cities, and the production of symbolic forms", Transactions of the Institute of British Geographers, 26 (13), Royal Geographical Society, London, pp. 11-23.

Scott, J. Allen (2004), “Cultural-products industries and urban economic development, prospects for growth market contestation in global context", Urban Affairs Review, 39 (4), Sage Journals, Thousand Oaks, pp. 461-490.

Scott, Allen (2006), "Creative cities: conceptual issues and policy questions", Journal of Urban Affairs, 28 (1), The Journal of de Urban Affairs Asociation, University of Wisconsin, Milwaukee, pp. 1-17.

Scott, J. Allen (2008), "Resurgent metropolis: economy, society and urbanization in an interconnected world", International Journal of Urban and Regional Research, 32 (3), Wiley Blackwell Publishing, New York, pp. 548-564.

Scott, J. Allen (2010), "Cultural economy and the creative field of the city”, Geografiska Annaler: Series B, Human Geography, 92 (2), Wiley, Suecia, pp. 115-130.

Solow, Robert (1956), "A contribution to the theory of economic growth", Quaterly Journal of Economics, 70 (1), Oxford University Press, Oxford, pp. 65-94. 
Storper, Michael y Richard Walker (1989), The capitalist imperative. Territory, technology and industrial growth, Blackwell Publishers, New York.

Storper, Michael (2005), "Society, community and economic development", Studies in Comparative International Development, 39 (4), Springer, London, pp. 30-57.

Swan, W. Trevor (1956), "Economic growth and capital accumulation", Economic Record, 32 (2), Wiley, Hoboken, pp. 334-361.

Throsby, David (2001), Economía y Cultura, Cambridge University Press, Cambridge.

Throsby, David (2008a), "Modeling the cultural industries", International Journal of Cultural Policy, 14 (3), Routledge, Taylor and Francis Group, London, pp. 217-232.

Throsby, David (2008b), "The concentric circles model of the cultural industries", Cultural Trends, 17 (3), Routlege, London, pp.147164.

unctad (Conferencia de las Naciones Unidas sobre Comercio y Desarrollo) (2009), "Portal de Desarrollo Económico y comercio", $<$ http://www.cinu.org.mx/temas/desarrollo/desecon/comercio. htm>, 9 de diciembre de 2009.

UnCTAD (Conferencia de las Naciones Unidas sobre Comercio y Desarrollo) (2013), "Trade in creative products reached new peak in 2011, UNCTAD figures show", http:/unctad.org/en/pages/ newsdetails.aspx? OriginalVersionID $=498 \&$ Sitemap_x0020_ Taxonomy=UNCTAD\%20Home;\#1385;\#International Trade and Commodities;\#1547;\#Creative Economy Programme; \#1851;\#Services, development and trade;\#1856;\#UNCTAD GSF 2013, 25 de mayo de 2014.

unESCO (Organización de Naciones Unidas para la Educación, la Ciencia y la Cultura) (2009) Portal Cultura, <http://portal.unesco.org/ culture/en/ev.php- URL_ID $=35257 \& \mathrm{URL}_{-} \mathrm{DO}=\mathrm{DO}_{-}$ TOPIC\&URL_SECTION=201.html>, 5 de octubre de 2009. 
unESCO (Organización de Naciones Unidas para la Educación, la Ciencia y la Cultura) (2013), “Creative Economy Report 2013” Special Edition, Widening Local Development Pathways, United Nation Development Program (UNDP) United Nations EducationalScientific and Cultural Organization (unEsCo), Paris, pp. 1-185.

Recibido: 20 de julio de 2012. Reenviado: 4 de diciembrede 2012. Aceptado: 23 de abril de 2013.

Ezequiel Avilés-Ochoa. Mexicano. Economista con doctorado en ciencias sociales por la Universidad Autónoma de Sinaloa. Actualmente es profesor e investigador de la Universidad de Occidente unidad Culiacán. Es miembro del Sistema Nacional de Investigadores, nivel II. Su línea de investigación actual es crecimiento y convergencia económica regional. Entre sus últimas publicaciones destacan: "El nivel básico de progreso sostenible. Una propuesta de categoría y metodología de medición”, en Mariano Rojas (coord.), La medición del progreso y del bienestar. Propuestas desde América Latina, Foro Consultivo Científico y Tecnológico, A.C., México, pp. 361-364 (2011); en coautoría, El capital humano en el desarrollo económico de Sinaloa, Gobierno del estado de Sinaloa y Conalep, Sinaloa (2009); Crecimiento económico y convergencia. Teoría y evidencia empirica, México, 1970-2004, Universidad de Occidente e Instituto Sinaloense de Cultura, Sinaloa (2008); Crecimiento y política económica. Sinaloa frente a México, Universidad de Occidente-Universidad Autónoma de Sinaloa-Dirección General de Fomento a la Cultura de Sinaloa, Sinaloa (2006).

Paola Marbella Canizalez-Ramírez. Mexicana. Es maestra en ciencias en estudios de América del norte por la Universidad Autónoma de Sinaloa. Actualmente se desempeña como asistente de investigación en el Departamento de Investigación y Posgrado de la Universidad de Occidente y coordina el Programa Educativo de la maestría en administración, unidad Culiacán. Su línea de investigación actual es desarrollo económico regional, particularmente, el proyecto de Industrias Culturales de Toronto. Entre sus publicaciones destacan, en coautoría: "El cluster de la manufactura alimentaria de Culiacán”, Sinaloa causa común, 3 (24), Coordinación General de Asesoría y Políticas Públicas, Sinaloa, pp. 6-9 (2007), en coautoría, "Industria de Alimentos", Ensayos sobre el desarrollo económico regional de Sinaloa, Universidad Autónoma de Sinaloa-Instituto Sinaloense de Cultura, Sinaloa, pp. 85-93 (2009). 